\title{
X-ray Crystallography of LpxB, a Glycosyltransferase in the Lipid A Synthesis Pathway
}

Thomas Bohl, John Lee, Ke Shi \& Hideki Aihara

Lipid A is the membrane anchor of lipopolysaccharide (LPS), the major component of the outer leaflet of the Gram negative outer membrane. LPS helps protect Gram negative bacteria from hydrophobic toxins, such as many antibiotics, thereby contributing to the growing problem of antibiotic resistance. In addition, lipid A (endotoxin) can overstimulate the innate immune system during sepsis resulting in septic shock. Sepsis causes 200 thousand deaths annually in part due to the toxic effects of lipid A. Therefore, the lipid A synthesis pathway is an attractive target for the development of new broad spectrum antibiotics that could be used to treat sepsis and other resistant bacterial infections. LpxB is the last highly conserved enzyme in the lipid A synthesis pathway and one of the two highly conserved enzymes of the lipid A pathway that have not been structurally characterized. Thus, the aim of this project has been to obtain crystal structures of $\operatorname{LpxB}$ and to model substrate binding. High resolution structures of solubilized LpxB were obtained bound to the substrate analogue $\mathrm{N}$-acetyl-glucosamine and in the apo state. LpxB forms two Rossmann-like domains as is typical for the Glycosyltransferase B family; however, LpxB forms a unique dimer with the C-termini of the polypeptides swapped between the subunits.

$\begin{array}{ll}\text { Thomas Bohl } & \text { University of Minnesota } \\ \text { John Lee } & \text { Bristol-Myers Squibb } \\ \text { Ke Shi } & \text { University of Minnesota } \\ \text { Hideki Aihara } & \text { University of Minnesota }\end{array}$

